\title{
NOTAS PARA LA CARRERA DOCENTE EN LA REPÚBLICA DOMINICANA
}

Career Notes for Teachers in Dominican Republic

\section{Celeste Abréu Van Grieken}

Es justo recibir según lo que nos corresponde y merecemos. Es justo asumir las consecuencias de nuestros actos y omisiones. Es justo que se nos reconozca y recompense cuando hacemos las cosas bien. Es justo defender nuestros derechos y los de los otros.

Urbieta

Resumen: Las notas que se presentan en este artículo inician con el estado actual de la profesión docente en la República Dominicana y sitúa la carrera docente, caracterizándola desde el marco legal. Asimismo, presenta una visión sistémica de esta carrera, considerando la diversidad de necesidades del ciclo de vida profesional docente. Finaliza planteando algunas ideas para la construcción de un Sistema de Carrera Docente desde un modelo más horizontal que evite la fuga de los mejores docentes de las aulas como vía para avanzar en la mejora del desempeño docente y la calidad de los aprendizajes de los sujetos.

Palabras claves: Formación inicial, desarrollo profesional, inserción docente, carrera docente, certificación, transdisciplinar. 
Abstract: This article begins with a presentation of the current status of the teaching profession in the Dominican Republic and positions such activity with a characterization from a legal point of view. This article also presents a systemic view of this career, taking into consideration the diversity of life-cycle needs of teachers. It ends raising some ideas for building a Teaching Career System from a more horizontal model that prevents the runaway of the best teachers from classrooms, as a way to further improve teacher's performance and subject's quality learning.

Keywords: Initial training, professional development, teaching inclusion, teaching career, certification, transdisciplinary.

\section{Estado actual de la profesión docente en la República Dominicana}

A pesar de los esfuerzos realizados a nivel oficial para la titulación de los y las docentes de la República Dominicana y de cursos de formación continua, la realidad es que han tenido muy poco impacto en las prácticas docentes y, por tanto, en la calidad de los aprendizajes de los sujetos. Los resultados de las pruebas nacionales y los estudios internacionales nos colocan en los últimos lugares entre los países de la región. En efecto, de acuerdo con el Informe de Progreso Educativo 2010, "Republica Dominicana es uno de los países con el peor desempeño estudiantil” (PREAL, 2010: 21).

Esta realidad no es sorprendente si asumimos que los y las docentes desempeñan un rol de gran importancia social como mediadores que facilitan los nuevos aprendizajes que en términos de competencia les exige la sociedad. De manera que estos sujetos educativos no solo deben ofrecer respuestas a las nuevas exigencias disciplinares y psicopedagógicas, sino atender las difíciles situaciones relacionales que surgen entre los estudiantes como resultado de la violencia intrafamiliar y social, la exclusión, la pobreza, el desempleo, la crisis económica 
en general. En ese sentido, es pertinente reconocer que la educación no está exenta de las problemáticas del contexto "pues la escuela a pesar de su inercia y rutina, aparece como una caja de resonancia de las contradicciones y paradojas de la sociedad en la cual está inserta" (Solar y Gatica, 2010: 148).

De ahí que ante la diversidad de roles que han de desempeñar estos sujetos educativos hay una agenda pendiente de grandes desafíos que afectan su práctica cotidiana, repercuten en la calidad de su desempeño y, por tanto, hay que abordarlos desde una perspectiva de políticas integrales.

En el intento de presentar una caracterización sucinta de los rasgos que a nuestro juicio configuran el estado actual de la profesión docente podemos señalar, la baja inversión gubernamental ${ }^{1}$ en el sector educación, la poca rigurosidad por parte de las instituciones formadoras para el acceso a los estudios de educación, los bajos perfiles de quienes optan por entrar a esta carrera, el desprestigio de la profesión docente, la feminización, las deficientes condiciones laborales; en la mayoría de los casos formación inicial deficiente por lo que las intervenciones docentes se guían más por la intuición que por los conocimientos adquiridos durante esa formación; condiciones precarias y en ambientes escolares poco acogedores y con escasez de recursos didácticos; jornadas laborales de más de una tanda para compensar los bajos salarios que reciben; la ausencia de un verdadero acompañamiento a los docentes en servicio así como de programas de inserción docente, las discontinuidades entre la formación inicial y la continua, la cultura del aislamiento docente y de una perspectiva de innovación permanente, falta de herramientas para asumir una actitud investigativa en el aula, una mal entendida autonomía del trabajo docente y la falta de sistematización y de rigor en la evaluación docente, entre otros factores.

\footnotetext{
${ }^{1} \mathrm{La}$ inversión hasta el momento ha sido menos del 3\% a pesar de que la ley establece un 4\% del Producto Interno Bruto para la Educación. Se espera que en la nueva gestión 2012-2016 se cumpla con esta disposición de manera que se puedan invertir esos recursos en las áreas del sistema que tienen mayores necesidades.
} 
Un rasgo que nos parece muy relevante al respecto es el referido a la realidad de la formación docente la cual no está diseñada como un continuo, es decir, no supera las fragmentaciones entre la formación inicial y la continua o desarrollo profesional, y articulada con la carrera docente. Precisamente el diagnóstico más reciente de la formación docente realizado en el año 2010 por el Ministerio de Educación Superior, Ciencia y Tecnología ${ }^{2}$ aporta datos significativos sobre las deficiencias de esta formación que convertidos en desafíos ameritan atención inmediata por parte de las instituciones formadoras y del ministerio correspondiente.

La lectura de esta realidad nos permite concluir que el estado actual de la profesión docente está en crisis y que necesitamos realizar esfuerzos sistemáticos en muchos órdenes para fortalecer una profesión que, como la docente, ha sido reconocida como una de las más importantes en términos del desarrollo sostenible de los pueblos.

\section{La carrera docente en la República Dominicana y otras visiones}

De acuerdo con la ley (66-97 General de Educación de la República Dominicana), en su Cap. 3, Art.135: "Se entiende por carrera docente la vinculación del servidor de la educación al conjunto de disposiciones organizativas y legales que regulan el ingreso, la permanencia, la promoción y el retiro de los docentes durante su ejercicio profesional; así como sus deberes y sus derechos laborales. La carrera docente comprende también el conjunto de disposiciones atinentes a la clasificación y valoración de cargo, el reclutamiento, la selección, el nombramiento de personal, la estabilidad, y evaluación del personal que brinden a la educación los mejores recursos humanos".

2 Cabe destacar la propuesta de políticas de formación docente formulada por este Ministerio, así como los estándares de formación, que de aplicarse constituirían un aporte relevante a la carrera docente. 
Esta misma ley establece, al igual que el Reglamento del Estatuto Docente, las condiciones de ingreso en las diferentes modalidades y niveles del sistema educativo. Asimismo, en el reglamento indicado, se define el régimen legal que clasifica los y las docentes por categorías y especialidades a partir de una escala de puntuación que privilegia la titulación. Sin embargo, en vista de que la experiencia demuestra muy poca relación entre la acumulación de títulos y la calidad del desempeño docente debe contemplarse la revisión de esos puntajes, incluyendo el establecimiento de categorías docentes que otorguen incentivos relacionados con la evaluación del desempeño, la innovación educativa, la producción bibliográfica, publicación de artículos, la investigación-acción y desempeños de calidad, entre otros criterios.

De todas maneras, aunque la carrera docente en el país ha sido conceptualizada desde esa visión de integralidad, en la práctica, la verticalidad en el ascenso es el criterio predominante con lo cual posiblemente, por razones de incentivos de los cargos, se alejan del aula los y las docentes que han adquirido una mejor formación y que muestran mejores niveles de desempeño.

Asimismo, se premia la antigüedad más que las habilidades y competencias docentes. Consecuentemente esta paradoja estimula el credencialismo, distorsionando el sentido de la formación y los niveles motivacionales requeridos para alcanzar la excelencia docente.

Por otra parte, en sentido general, el concurso es la modalidad de acceso a la carrera docente, aunque hasta el momento solo se aplica al nivel básico con no muy buenos resultados, pues se han puesto en evidencia las debilidades formativas de la mayoría de los docentes que participan en las convocatorias realizadas con esos fines. En ese sentido, cabe destacar que "el sistema de concursos para acceder a los cargos docentes requiere de objetividad y transparencia junto con una asignación de plazas docentes acorde con las características específicas de las escuelas" (PREAL, 2011: 51). 
Otros modelos de carrera docente con una orientación más horizontal van marcando el sendero en la región con mayores niveles de descentralización, así como de participación y toma de decisiones de los docentes en aspectos relativos al desarrollo curricular y el otorgamiento de incentivos a partir de la evaluación del desempeño sin que implique dejar el aula en la búsqueda de mejores condiciones salariales.

Esta mirada de la carrera docente está "más ligada al desarrollo profesional del docente, a su formación continua, que estimule la adquisición de nuevas capacidades y conocimientos, en el marco de mecanismos institucionales que promuevan la salida transitoria del puesto de trabajo frente a alumnos, para capacitarse o para participar de experiencias de innovación o investigación, y retornar luego al trabajo en el aula en condiciones superiores de formación" (Terigui, 2010: 27).

Asimismo, esos modelos "intentan buscar la mejora de los resultados de calidad educativa, la retención de los alumnos y mayores niveles de equidad como principales objetivos. La intención es trasladar esos objetivos al modelo de incentivos de las carreras y procurar que los docentes los vivan como propios." (Laies y Santos, 2010: 5).

Por tanto, estamos abocados a una nueva visión de esta carrera para que podamos revertir los efectos perversos señalados respecto de la situación actual y avanzar hacia una carrera que no solo asegure la permanencia en el sistema educativo, sino que sea reconocida socialmente como una profesión de prestigio a la cual los mejores talentos quieran pertenecer. 


\section{Desarrollo de la profesión docente desde una visión sistémica de la carrera docente}

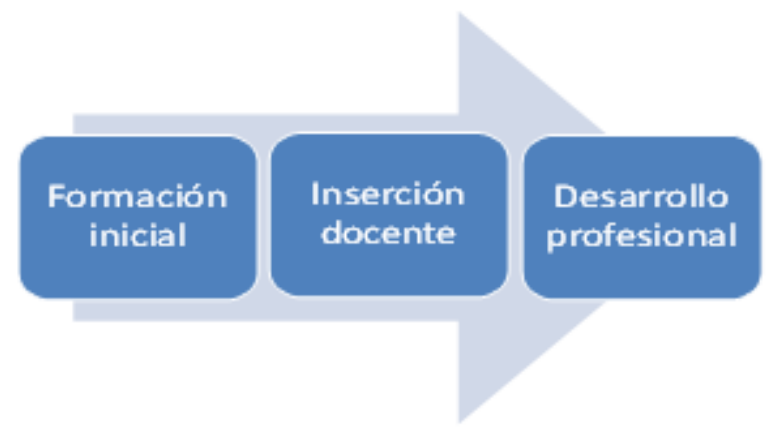

\section{a) FORMACIÓN INICIAL}

Convertir la carrera docente en una profesión atractiva que atraiga y retenga los mejores talentos como en otros países, Finlandia y Suecia por citar dos casos, constituye una legítima aspiración de todos los países que aspiran la mejora de sus sistemas educativos. ${ }^{3}$ Obviamente, en la República Dominicana con una profesión mal valorada por la sociedad en su conjunto, dado el pobre desempeño docente, resulta muy difícil atraer a los mejores estudiantes a la docencia a menos que se produzca un cambio en las políticas educativas desde todos los subsistemas que conforman el sistema educativo en su conjunto, es decir, desde una visión sistémica.

De acuerdo con Flores (2008), en la República Dominicana, la mayoría de los estudiantes que ingresan a los estudios de educación proceden de sectores humildes y con serias limitaciones académicas y culturales. Posiblemente el facilismo en las condiciones de acceso

\footnotetext{
${ }^{3}$ Cabe destacar los esfuerzos realizados por el Instituto Nacional de Formación y Capacitación del Magisterio (INAFOCAM) que recientemente editó un vídeo con la intencionalidad de motivar y atraer esos talentos desde la perspectiva de los aportes que pueden hacer los docentes al país, destacando además los beneficios que ofrece el sistema educativo a los docentes.
} 
y la falta de rigor académico en muchos casos hayan producido una cantidad de egresados de la carrera de educación con una formación débil lo cual impacta de manera directa los aprendizajes de los sujetos. Asimismo las discontinuidades entre la formación inicial y la continua o desarrollo profesional y de un acompañamiento sistemático afectan sensiblemente la calidad del desempeño docente. Esta realidad es, en consecuencia, uno de los desafíos pendientes si queremos una formación cuyo impacto en las aulas revierta los resultados de aprendizajes obtenidos hasta el momento.

Otro factor importante que debe considerarse en la formación inicial es el referido a la formación de los formadores de formadores. ¿Cuáles son las diferencias metodológicas que aportan estos formadores respecto de la formación recibida a nivel medio y a nivel superior?

En lo referente a la formación de los formadores se requieren estrategias que posibiliten un cambio en sus concepcionesy, por tanto, en las metodologias que utilizan en la formación docente, pues "la escasa evidencia empirica de la que se dispone, muestra que los formadores tienden a utilizar un estilo de enseñanza frontal similar al que recibieron tanto en educación básica como en su formación profesional" (Vaillant, 2004: 23).

Además en términos curriculares y de uso de recursos, ¿qué tipo de articulación realizan estos formadores entre teoría-práctica? ¿Qué experiencias de investigación-acción proporcionan a los futuros docentes que les permita desarrollar una perspectiva indagadora que posibilite la problematización de su práctica, desarrollar una actitud autocrítica, reflexiva, y de innovación? ¿Cuáles estrategias emplean para superar la visión fragmentada del conocimiento y establecer las conexiones inter y transdisciplinarias que se requieren? ¿Qué aportes hace esta formación a la perspectiva de género? ¿Cuál es el uso que se hace de las tecnologías de la información y comunicación a fin de que puedan incorporarlas como parte esencial de su autoformación y de su desempeño? ¿Qué experiencias de formación para la vida ético-ciudadana incorporan a todo el proceso formativo de estos 
docentes? ¿Cuál puede ser el resultado de una formación docente que no reconozca las vinculaciones realidad-escuela como parte fundamental de su misión?

En ese sentido, habría que abocarse a la superación de modelos formativos tradicionales que todavía mantienen a estos formadores en roles que no favorecen que los sujetos sean el centro del aprendizaje. Por ejemplo, que incluya desde el inicio de la carrera debates sobre el estudio de casos, grabaciones de las observaciones de clases con posteriores sesiones críticas y autocríticas, proyectos de trabajo interdisciplinarios, trabajo entre pares, biografías de vida como vía de desarrollo personal y de desarrollo de la inteligencia intrapersonal; uso de instrumentos que favorezcan la reflexión y que puedan generar un pensamiento docente desde una perspectiva crítica e innovadora, desde una metodología que favorezca la investigación-acción.

Dado que la metodología de la investigación-acción se desarrolla en una espiral, Car y Kemmis (1988: 197) señalan que requiere una epistemología distinta:

Implica tanto la intervención controlada como el juicio práctico, aunque ambos tienen atribuido un lugar en la noción de la espiral autorreflexiva de la investigación-acción que se dispone como un programa de intervención activa y de juicio práctico conducido por individuos comprometidos no solo a entender el mundo, sino también a cambiarlo.

Todas estas herramientas sin pretender que sean exhaustivas, pueden aportar nuevas maneras de intervención docente y constituir el portafolio individual que permita apreciar el carácter evolutivo de una práctica docente que se crea y se recrea constantemente, superando la rutina y la repetición.

Asimismo, existe la necesidad de promover en programas de maestrías para estos formadores, así como de programas de formativos intensivos coherentes con las características institucionales de la oferta programática de las instituciones formadoras de docentes, que no 
solo se diferencien de las metodologías empleadas en la educación media, sino que al constituirse en ejes de las vivencias formativas de los futuros profesionales de la docencia puedan integrarse a su práctica de manera natural y no como eventos excepcionales.

Finalmente, cabe destacar la necesidad de fortalecer la habilitación docente, una estrategia de la formación inicial dirigida a profesionales de diferentes áreas del conocimiento, quienes reciben una formación pedagógica en programas diseñados para tales fines que permitiría superar las debilidades que, a nivel de las diferentes disciplinas, presentan la mayoría de los y las docentes formados para el nivel medio.

\section{b) INSERCIÓN DOCENTE}

De acuerdo con el Estatuto Docente de la República Dominicana, se establece un año como período de prueba para los y las docentes que ingresan por primera vez al Sistema Educativo Dominicano, al final del cual se debe aplicar una evaluación de desempeño, sin embargo, esta disposición legal no se cumple.

En una consulta con expertos nacionales sobre la carrera Docente realizada en el 2010, en el Instituto Nacional de Capacitación y Formación Docente se consideró que ese período debía ampliarse a tres años con un acompañamiento sistemático y una evaluación final como está establecido en el citado reglamento.

Sin embargo, aunque se reconoce el impacto de estos primeros años de vida profesional e incluso la inserción docente ${ }^{4}$ ha sido planteada en el marco de los debates de la educación que queremos para el siglo XXI, en el país todavía no se han formulado políticas que regulen esa importante fase del ciclo de vida de los y las docentes. En definitiva, la inserción docente se considera un período crítico, no solo porque comprende las primeras experiencias de vida

\footnotetext{
${ }^{4}$ Preferimos el término inserción en lugar de inducción para referirnos a ese primer eslabón del ejercicio docente.
} 
profesional en las aulas, sino porque el mismo aislamiento docente y el no disponer de una estructura rigurosa de acompañamiento deja en el vacío a estos profesionales principiantes que posiblemente de no recibir el apoyo de pares más experimentados repiten el ciclo de la deficiente formación recibida. Por tanto, "si las experiencias iniciales de su primer nombramiento como docentes no les proporcionan una buena base en el contexto de estas nuevas orientaciones, es probable que utilicen sus propias experiencias de alumnos como plantilla para su propio trabajo.” (Knight, 2012: 55).

En la experiencia de otros países de la región, como Chile, Argentina y Colombia, solo por citar algunos existen políticas que proporcionan procesos de inserción a estos profesores principiantes de manera que les permita superar los temores y ansiedades de esta primera etapa de su ciclo profesional al tiempo que les permite desarrollar seguridad en su desempeño, así como habilidades y competencias para resolver las situaciones que la vida del aula les va planteando.

En general, la bibliografía consultada al respecto considera que "la inducción de los profesores debe ser un proceso guiado por docentes con alta experiencia y experticia en el proceso de enseñanzaaprendizaje, a partir de los cuales los "neófitos" puedan aprender y formarse en la práctica, y así, ir asumiendo progresivamente mayores tareas, responsabilidades y funciones dentro del aula y del establecimiento.” (CPUC, 2012; 17).

Actualmente, el Ministerio de Educación en coordinación con el Instituto Nacional de Formación y Capacitación Docente y el apoyo de la Organización de Estados Iberoamericanos OEI están dando los primeros pasos para llevar a cabo en el país una experiencia piloto con los docentes principiantes que, a partir de los aprendizajes generados, permita formular políticas al respecto. ${ }^{5}$

\footnotetext{
${ }^{5}$ Estas instituciones publicaron una Guía para la Inserción de Docentes Principiantes, y organizaron un proceso formativo sobre acompañamiento a los posibles mentores, pero aún no se ha iniciado el programa piloto diseñado para tales fines.
} 


\section{C) DeSARRollo PROFESIONAL}

En el marco de las Mesas Consultivas que se conformaron como espacio de debate y acuerdos para las publicaciones Metas Educativas 2021 se señala lo siguiente:

La necesidad de vincular, en la práctica el Estatuto de la Carrera Docente a un programa de desarrollo profesional ha sido expresada de manera reiterada en el ámbito educativo dominicano desde hace más de una década. Se aboga por un modelo donde la formación inicial, la inducción y la formación continua se constituyan en un itinerario común para todos los docentes del Sistema (2010: 152).

Desde la perspectiva de ese desarrollo profesional, se aprecia el intento de pasar desde una visión técnico-pedagógica del docente a una visión profesional como un sujeto con autonomía y capacidad para tomar decisiones, con criterio para manejar el currículo y recrearlo, con sentido de misión y vocación, conducta ética en su vida personal y profesional, con habilidades para auto gestionar su formación, trabajar en equipo, reflexionar sobre su práctica, sistematizarla y compartirla con otros pares.

¿Cómo realizar el tránsito hacia esa visión de la profesionalización docente? Nos parece que aunque no hay recetas, tienen pertinencia para cualquier contexto los planteamientos de Hubermam (1999: 72) quien expresa que:

Las actitudes y las prácticas de los enseñantes deben llegar a estos más profundamente ancladas en un fundamento teórico y de investigación educativa; la autonomía profesional de los maestros debe ampliarse en el sentido de incluirlos en las decisiones que se tomen sobre el contexto educacional más amplio dentro del cual actúan; es decir, que la autonomía profesional debe ser respetada tanto en el plano colectivo como en el individual. 
Coincidimos con estas aportaciones, pues el trayecto hacia la aspirada profesionalización de los y las docentes no es posible alcanzar, sin que tengan una participación activa en la toma de decisiones y un empoderamiento en la variada gama de nuevos roles que deben desempeñar.

Ese desarrollo profesional también requiere un conocimiento profundo de las verdaderas necesidades formativas del personal docente, a fin de que la articulación entre la formación inicial y la continua se lleve a cabo desde una dinámica de cambio e innovación que motive al personal docente a mantener la curiosidad científica en su área disciplinar y en lo pedagógico y, por tanto, el deseo de seguir aprendiendo durante toda su vida.

Asimismo, esas necesidades han de repensarse desde la concepción de la escuela que aspiramos: Una escuela donde no solo aprendan los estudiantes, sino todos los sujetos educativos. Entre esos sujetos, los y las docentes, los equipos de gestión y de acompañamiento tienen en las aulas espacios ideales para crear y recrear la actuación docente; para dar a conocer lo que hacen, interpelar sus prácticas, identificar situaciones que requieren investigación-acción, crear un clima de revalorización y construcción de los saberes docentes que fortalezca la autoestima docente, así como la movilización permanente de su imaginario profesional.

Por tanto, la dimensión pedagógica de los equipos de gestión de las escuelas debe incluir como parte de su proyecto institucional o curricular espacios destinados a la formación en y desde el centro educativo, considerando las necesidades formativas identificadas durante los procesos de acompañamiento.

En lo relativo a los incentivos que aporta la perspectiva de desarrollo profesional docente a los procesos motivacionales, es importante destacar los aportes de Terigui sobre el tipo de estímulos que deberían contemplar con relación a la carrera docente (2009: 93): 
La aprobación en un curso desafiante y su aprobación; la elaboración de un proyecto a realizar en la escuela. Su puesta en marcha y aprobación; la satisfacción de ganar un concurso calificado y el prestigio que eso conlleva; la identificación de campos problemáticos en la institución escolar y la construcción de dispositivos que favorezcan la búsqueda de soluciones; la presentación a becas de estudio; el asesoramiento a un colega que se inicia son ejemplos de importantes logros para los docentes que incorporados en la perspectiva de la trayectoria laboral, pueden estructurar una carrera profesional atractiva y dotada de estimulos.

¿No serán estos estímulos una manera de promover la reiterada autonomía profesional que aspiramos para nuestros docentes?

Por otra parte, también ha de considerarse la relevancia que tiene para los y las docentes el sentido de misión inherente a la naturaleza de la función formativa que realizan. De ahí la necesidad de replantearse esa mirada del desarrollo profesional considerando la dimensión humana de estos sujetos.

Entre las diferentes propuestas sobre el sentido del desarrollo profesional docente y su incidencia en esa profesionalización, destacamos la aportada por Day (2006: 145) quien señala la que la mejora de la escuela requiere un tiempo a este desarrollo. Asimismo, apunta cómo la mejora de los y las docentes debe situarse en el contexto de las necesidades personales e institucionales las cuales no siempre coinciden. Por último, expresa que "el corazón de los docentes (pasiones, entusiasmo, identidad personal, compromiso, emociones) es un centro de atención tan importante como su cabeza y sus manos.

Cabe preguntarse, en las condiciones actuales de la formación docente y las precariedades existentes en la escuela y de las actuales condiciones laborales, ¿cómo estará el corazón de la mayoría de los y las docentes dominicanos? ¿Cómo asumir con pasión un quehacer que está permeado por valores, emociones, sentimientos y al mismo tiempo realizado en condiciones generalmente críticas para la sobrevivencia? 


\section{Articulaciones necesarias para un Sistema de Carrera Docente en República Dominicana}

¿Cómo lograr el tránsito hacia la concepción de una carrera docente que permita la formulación y aplicación de políticas integrales consensuadas que mejoren y dignifiquen las condiciones actuales de los y las docentes?

En el contexto de las necesidades actuales y de futuro de la República Dominicana nos parece pertinente considerar, sin pretender ser exhaustivos:

a) La necesidad de articular la carrera docente a los procesos formativos de los y las docentes como un continuo, sin fragmentaciones carentes de significado para el trabajo en las aulas.

b) Formular políticas que permitan y favorezcan la tanda única con espacios destinados a trabajos entre equipos docentes para planificar, coordinar procesos del desarrollo curricular e intercambiar experiencias docentes con otros pares como parte de los procesos de formación desde el centro educativo.

c) Las Políticas de Desarrollo Profesional con el bienestar de los y las docentes desde una amplia visión que incluya no solamente aspectos económicos, sino de responsabilidad social y de orgullo por la identidad de una profesión que tiene una función liberadora y de transformación social.

d) Favorecer la responsabilidad por los resultados y una cultura de rendición de cuentas.

e) Aunque la evaluación del desempeño basada en estándares de desempeño es un tema conflictivo, debe realizarse en todos los tramos o categorías de la carrera docente y articularse a los 
incentivos $^{6}$ y a programas formativos que permitan superar las debilidades evidenciadas en esa evaluación.

f) Impulsar políticas de acreditación de carreras y programas que posibiliten asegurar la calidad de la formación docente inicial y de post grado. ${ }^{7}$

g) Las promociones y los ascensos de los docentes necesitan replantearse a partir de criterios más horizontales relacionados con categorías en el marco de un mismo cargo para evitar la fuga de los buenos docentes a puestos directivos en perjuicio de la calidad del trabajo en las aulas.

h) Aplicar el Tribunal de la Carrera Docente para velar por el cumplimiento de los deberes y derechos de los y las docentes.

i) Reorientar el sentido de misión del sindicato docente de manera que recupere el prestigio que marcó sus inicios.

j) Aplicar políticas de certificación y recertificación docente.

\section{Conclusión}

Dado que la carrera docente tiene tanta significación en la vida de los y las docentes, su reorientación hacia la calidad del desempeño es uno de los retos más importantes del Sistema Educativo Dominicano. Esa reconstrucción posibilitaría al docente una visión a largo plazo del itinerario de su vida profesional de acuerdo con sus proyectos de desarrollo. Asimismo, en función de los niveles de calidad que establezca el Sistema de Formación y de Carrera Docente, podría

\footnotetext{
${ }^{6}$ En la actualidad funciona el Instituto Nacional de Bienestar Magisterial (INABIMA) que ejecuta un conjunto de servicios para mejorar la calidad de vida de los maestros, como seguro dental y médico, servicios funerarios, pensiones y jubilaciones, entre otros.

${ }^{7}$ Recientemente, el Ministerio de Educación ha creado una oficina de Certificación para la Carrera Docente 2013 y se realiza un proceso consultivo para el monitoreo de las principales acciones programáticas definidas como metas parciales preparatorias.
} 
contribuir a transformar la autoimagen del docente y a superar la pobre percepción social que se tiene de la profesión docente.

Hay que procurar una carrera docente desde una perspectiva de políticas sistémicas que incluya la captación de los mejores talentos, mejorar los criterios de acceso a las instituciones formadoras, concretizar los procesos de inducción a docentes principiantes, articulación de estos procesos formativos con los incentivos correspondientes, las condiciones laborales y las experiencias de desarrollo profesional permanente, evaluación del desempeño y procesos de certificación y recertificación docente no solo proporcionaría estabilidad, seguridad y equilibrio en términos de los deberes y derechos de los y las docentes, sino la calidad del desempeño docente y, por tanto, de los aprendizajes de los sujetos.

\section{Bibliografía}

Carr, S. y Kemmis, W. (1988). Teoría y crítica de la enseñanza, Ediciones Martínez Roca, S.A., Barcelona.

Centro de Políticas Públicas UC (2012), Propuestas para una Carrera Docente. Ponficia Universidad Católica de Chile.

CONEA-CEAACES, (2011) Modelo General para la Evaluación de Carreras con fines de Acreditación. Ecuador.

Day, C. (2006). Pasión por enseñar. Narcea, S.A. de Ediciones, Madrid.

Flores, R. (2008). Estudio sobre formación de maestros y directores, Secretaría de Estado de educación, Santo Domingo.

Hubermam, S. (1999). Como se forman los capacitadores. Editorial Paidós, Buenos Aires.

Metas Educativas (2010). Consulta Nacional, Serigraf, S.A., Santo Domingo. 
Ministerio de Educación, Ciencia y Tecnología (MESCyT) (2010). Plan reformulación de la formación docente, Santo Domingo.

Laies y Santos (2010). Nuevos docentes, nuevas carreras. Centro de Estudios en Políticas Públicas, Buenos aires.

Ley General de Educación, Secretaría de Estado de Educación y Cultura (SEEC), 1997. Disponible en www.educando.edu.do. [Recuperado el 2 de octubre de 2012].

Reglamento del Estatuto del Docente. (2003). Secretaría de Estado de Educación, Santo Domingo.

Programa de Promoción de la Reforma Educativa en América Latina y el Caribe-PREAL- Informe de Progreso Educativo República Dominicana 2010, Santo Domingo.

Programa de Promoción de la Reforma Educativa en América Latina y el Caribe -PREAL-Construcción de la profesión docente en América Latina: Tendencias, temas y debates, Edición de Vaillant, D., 2004, Santiago de Chile.

Programa de Promoción de la Reforma Educativa en América Latina y el Caribe-PREAL-Estado del Arte sobre Politicas Docentes en América Latina y el Caribe. 2011.

Programa de Promoción de la Reforma Educativa en América Latina y el Caribe -PREAL- (2012). Desarrollo profesional continuo y carrera docente en América Latina. Edición de Flavia, T., Santiago de Chile.

Picón, C., Abréu, C., y Moreta, Y. (2010). Marco de referencia para la construcción de un Sistema de Carrera Docente que articula la formación docente y otros elementos. Documento inédito elaborado a solicitud del Instituto Nacional de Formación y Capacitación del Magisterio (INAFOCAM), Santo Domingo. 
Urbieta, R. (2005). El regalo de sí mismo, Narcea, S.A. de Ediciones, Madrid.

Terigui, F. (2009). Carrera docente y politicas de desarrollo profesional. Aprendizaje y desarrollo profesional docente. Metas educativas 2021. Medrano y Vaillant. Organización de Estados Iberoamericanos (OEI), Madrid.

Solar, S. y Gatica, N. (2010). Autonomía, heteronomía y educación. Educación, valores y ciudadanía. Metas educativas 2021. Organización de Estados Iberoamericanos (OEI), Madrid.

Knight, P. (2012). El profesorado de Educación Superior. (3ra. ed.), Narcea, S. A. de Ediciones.

\section{Celeste Abréu Van Grieken}

Es licenciada en Educación (UASD).

Tiene un Postgrado en Supervisión Educativa y Maestría en Educación (INTEC). Se desempeña como asesora académica del INAFOCAM y consultora de varios centros educativos privados. Ha publicado varios artículos relacionados con la enseñanza y la pedagogía moderna.

Email: abreuceleste@hotmail.com

Recibido: 10/10/2012 Aprobado: 15/11/2012 
